\title{
Disruption of the Brain Resting State Networks in Parkinsonism
}

\author{
Mahdieh Ghasemi", Ali Foroutannia
}

Department of Electrical Engineering, University of Neyshabur, Neyshabur, Iran

\section{ABSTRACT}

Introduction: In the recent years, neuroimaging research on functional Magnetic Resonance Imaging (fMRI) is used in many pathological and mental conditions. The analysis of alterations in the resting state networks (RSN) is an important method for the scrupulous understanding of the function and connectivity changes of the disease in order to provide new diagnostic and therapeutic approaches. In this paper, we studied the resting-state functional MRI (RsfMRI) data in Parkinson's disease (PD) to explore the complex disruption in the RSNs and the functional interactions between them. Materials and Methods: A total Rs-fMRI data of 10 Parkinsonism and 10 healthy people in the 3T-MRI system were considered. Probabilistic independent component analysis (PICA) was used to extract network components. RSNs were identified using spatial correlation with a rest reference template network. Dual regression and randomize technique calculated individual differences between the groups. Results: Group component maps resulted in some main clusters of RSN that significantly overlapped with the reference network, such as the visual cortex, salience network, and supplementary motor area. Individual differences between RSN maps identified temporal, salience and cingulate networks as the main clusters. Conclusion: Most of the previous studies investigated the functional connectivity alterations in PD by seed-based analysis. Here, we employed the datadriven approach based on group PICA to extract and evaluate RSN changes in all related neural networks. Our finding indicates that changes of the functional architecture of the RSNs are associated with PD.

\section{Key words:}

1. Magnetic Resonance Imaging 2. Neurodegenerative Diseases

3. Diagnostic Imaging

4. Brain

*Corresponding Author: Mahdieh Ghasemi

E-mail:m.ghasemi@neyshabur.ac.ir 


\section{اختلالات شبكه استراحت مغز در بيماران پاركينسونى}

\section{مهديه قاسمى"، على فروتننيا}

كروه مهندسى برق، دانشكاه نيشابور، نيشابور، ايران

كليد وازهها:

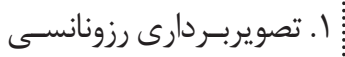
مغنا طيسى

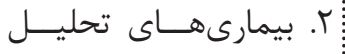

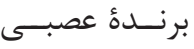

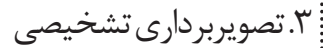
(f

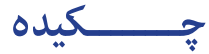

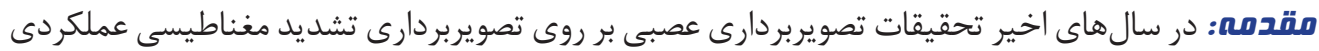

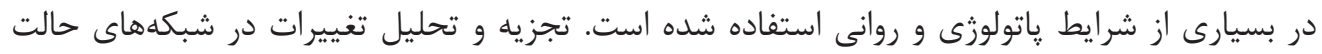

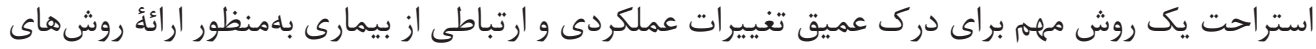

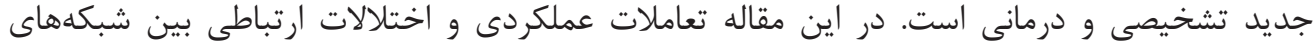

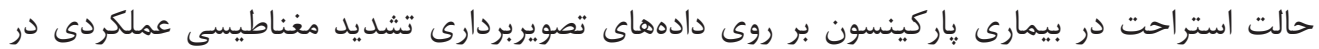

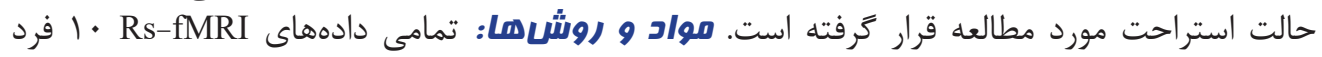

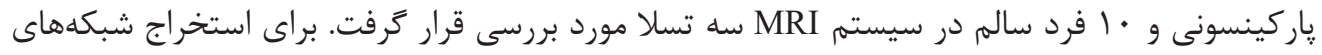

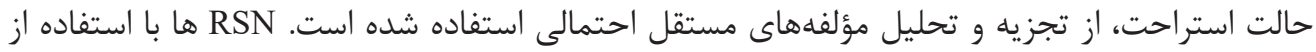

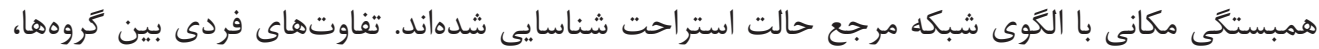

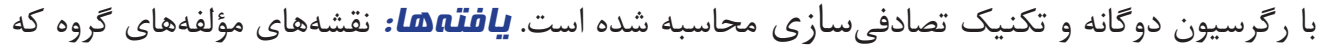

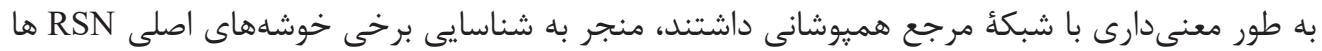

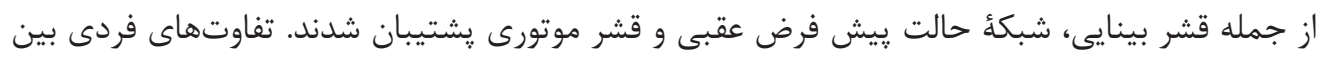

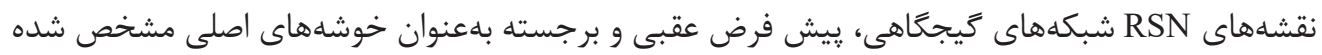

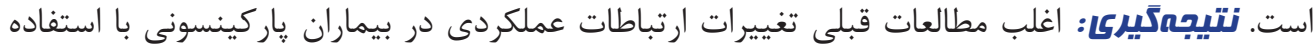

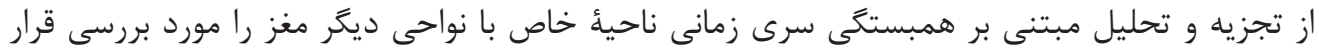

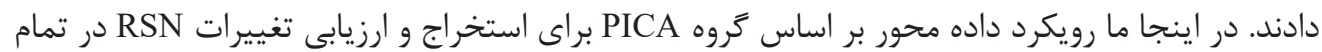

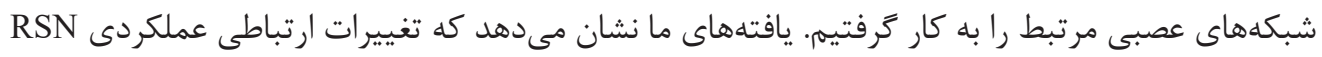

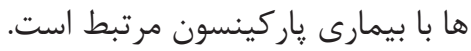




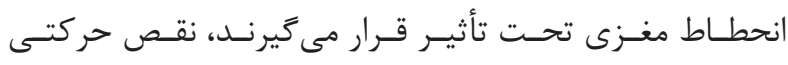

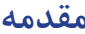

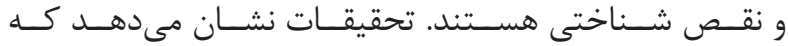

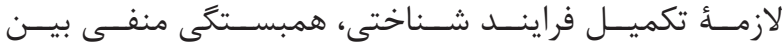

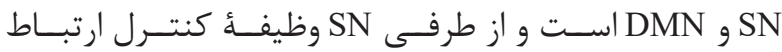

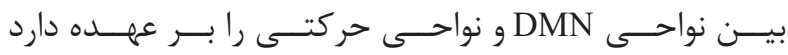

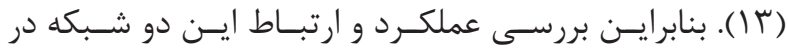

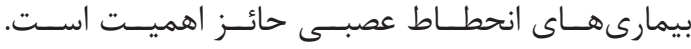

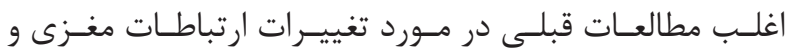

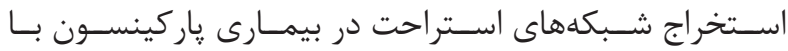

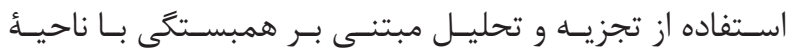

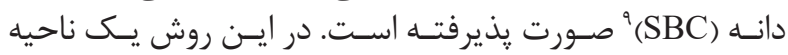

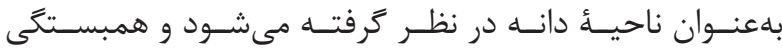

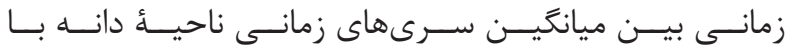

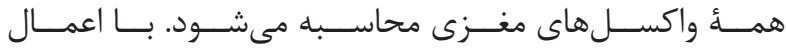

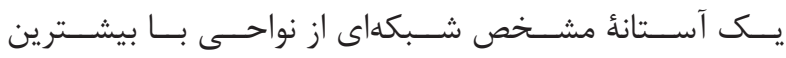

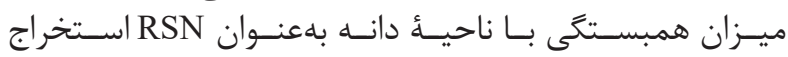

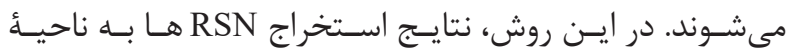

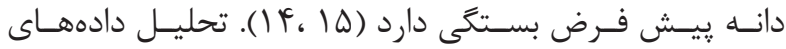
Rs-fMRI

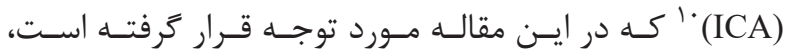

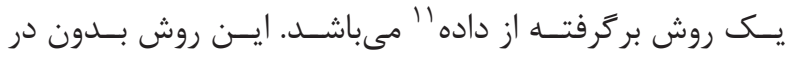

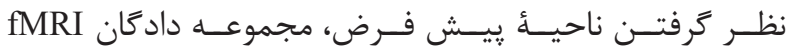

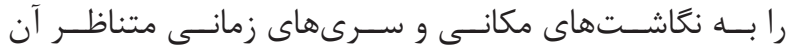

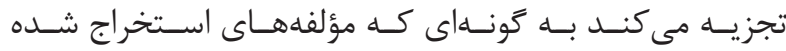

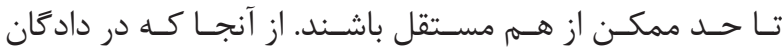

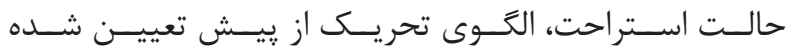

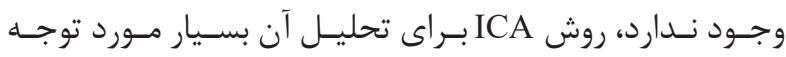

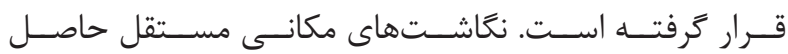

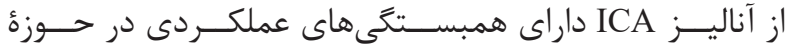

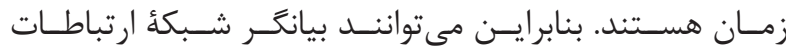

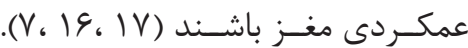

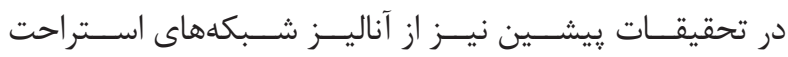

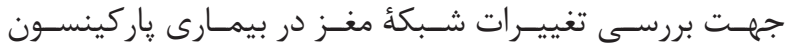

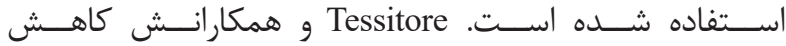

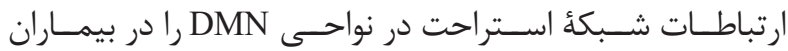

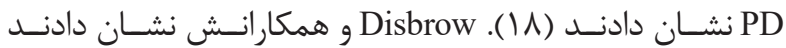

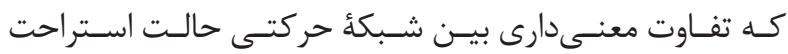

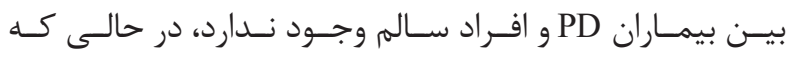

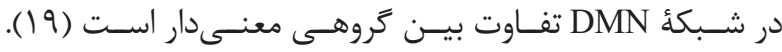

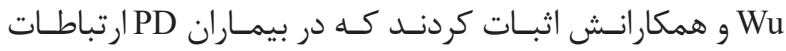

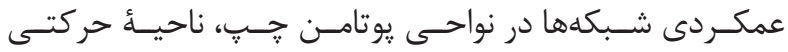

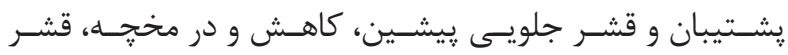

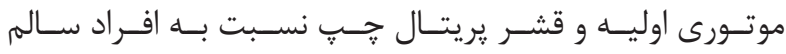

${ }^{1}$ Parkinson's disease

${ }^{2}$ Functional magnetic resonance imaging

${ }^{3}$ Blood oxygen level dependent

${ }^{4}$ Resting state functional magnetic resonance imaging

${ }^{5}$ Resting state network

${ }^{6}$ Salience network

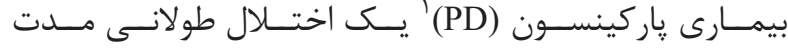

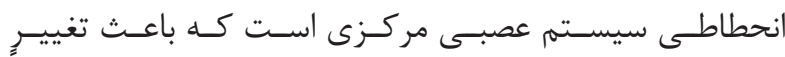

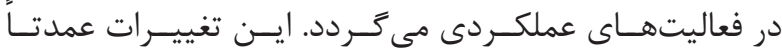

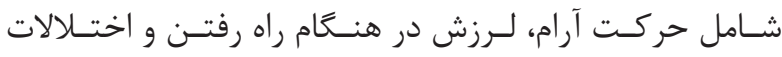

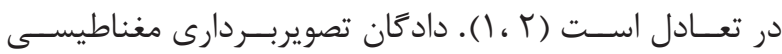

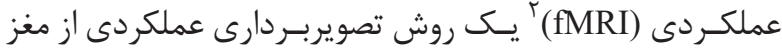

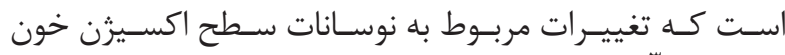
r (BOLD)

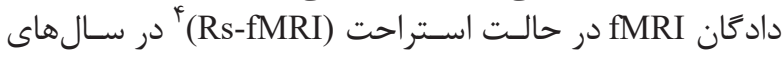

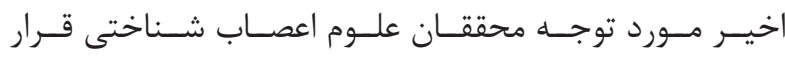

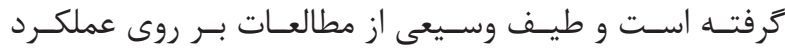

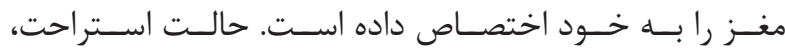

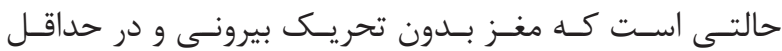

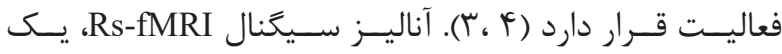

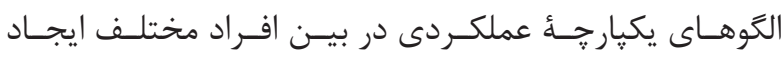

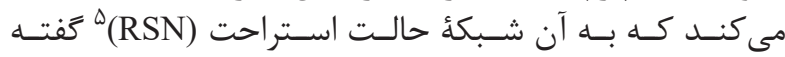

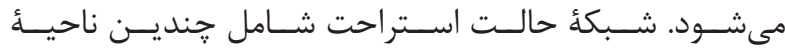

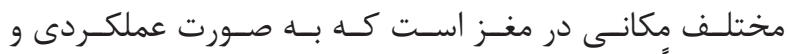

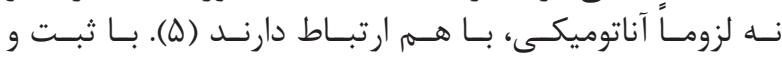

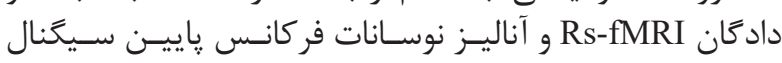

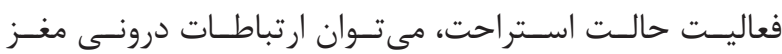

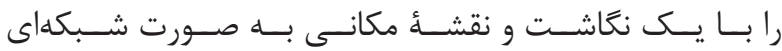

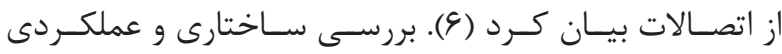

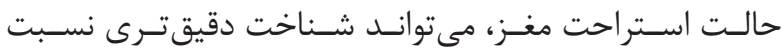

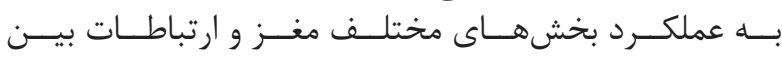
نواحسى مختلـف ايجـاد كنــد مختص

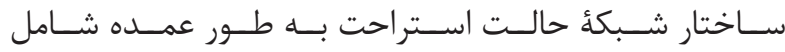

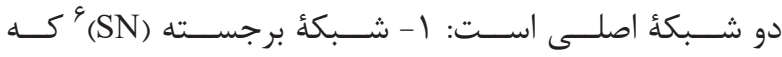

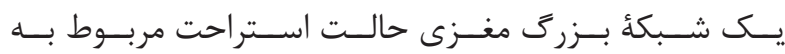

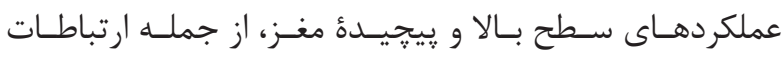

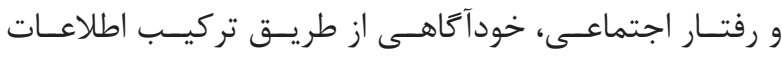

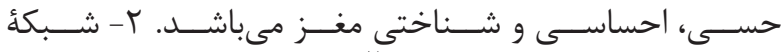

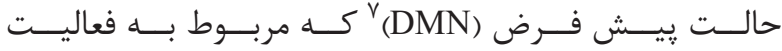

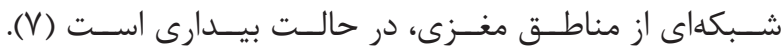

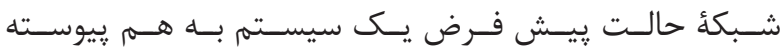

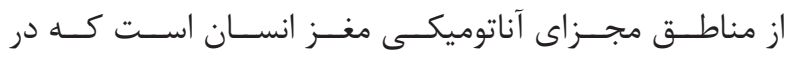

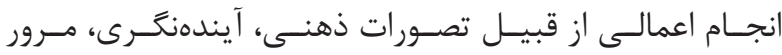

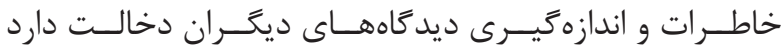

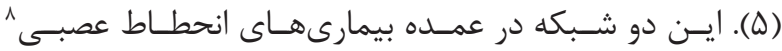

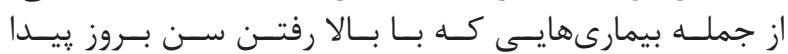

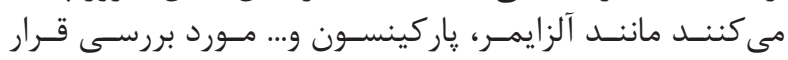

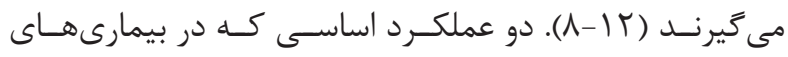

${ }^{7}$ Default mode network

${ }^{8}$ Neurodegenerative disorders

${ }^{9}$ Seed based correlation

${ }^{10}$ Independent component analysis

${ }^{11}$ Data driven 


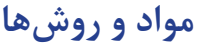

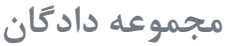

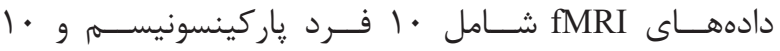

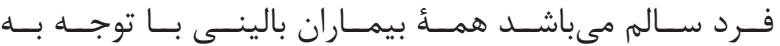

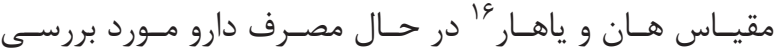

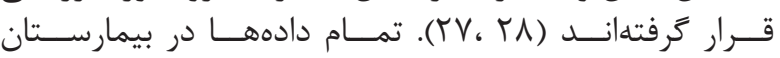

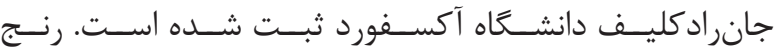

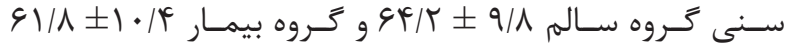

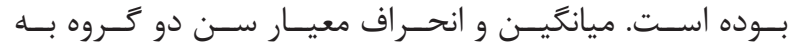

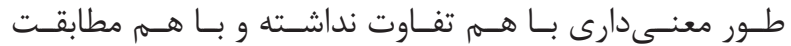

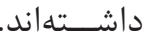

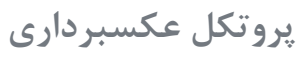

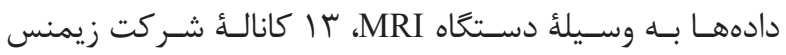

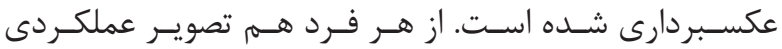

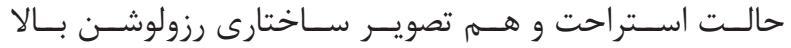

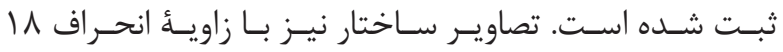

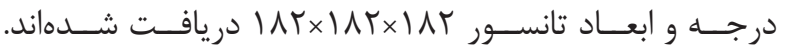

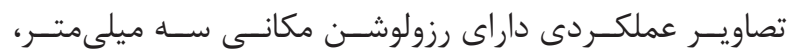

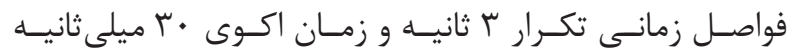

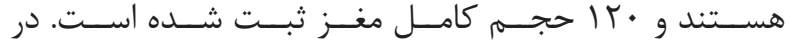

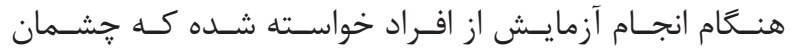

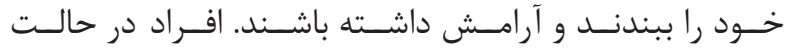
اســراحت و هوشــيار بودهانــد.

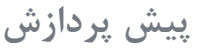

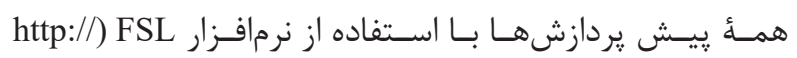

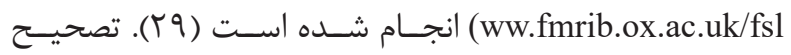

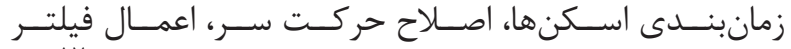

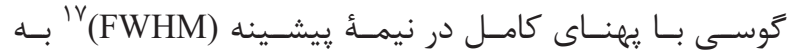

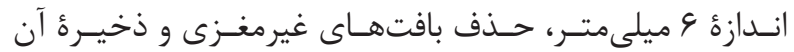

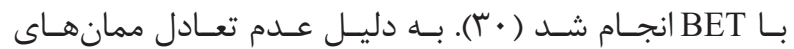

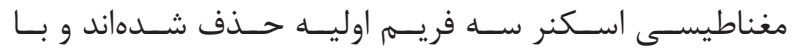

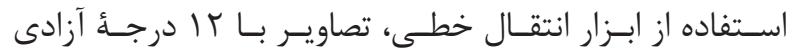

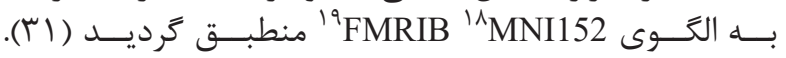

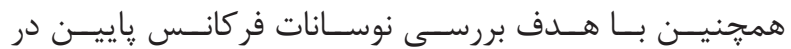

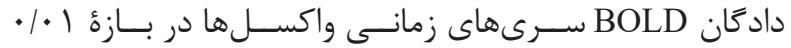

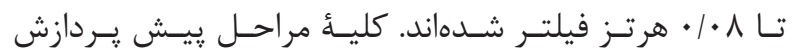

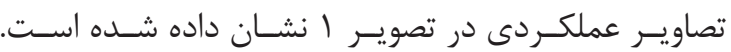

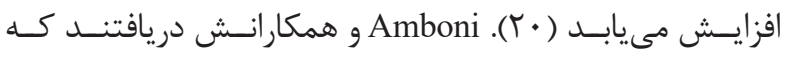

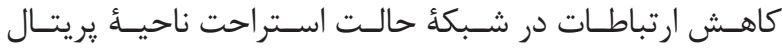

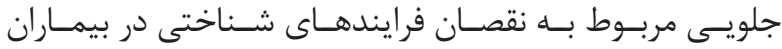

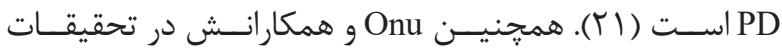

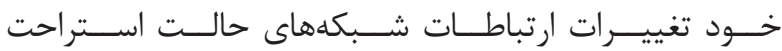

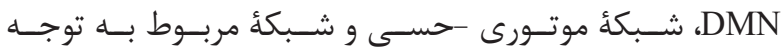

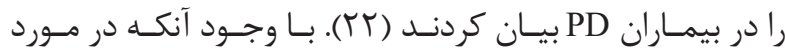

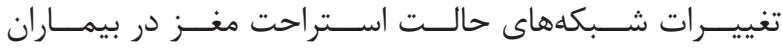

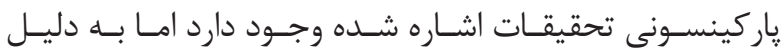

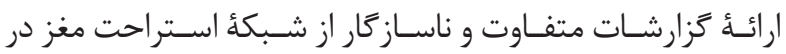

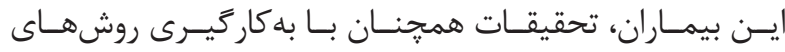

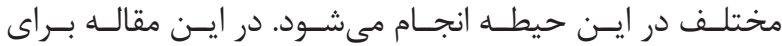

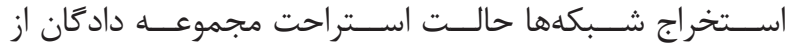

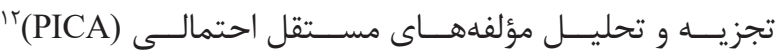

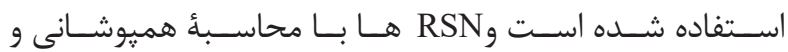

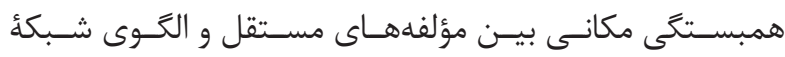

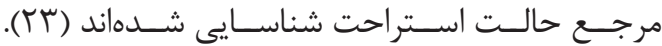

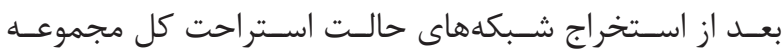

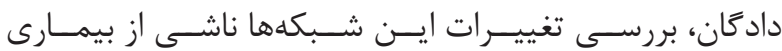

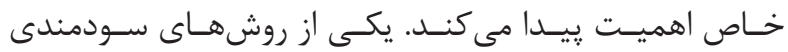

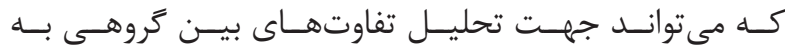

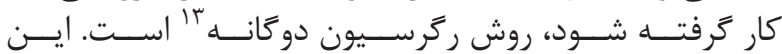

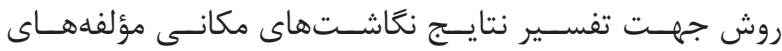

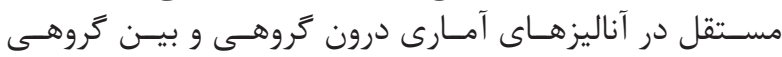

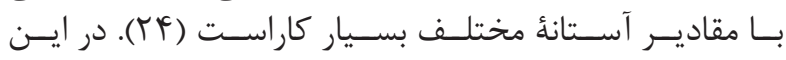

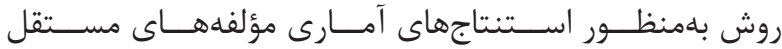

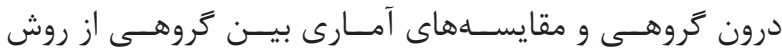

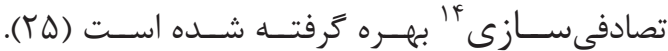

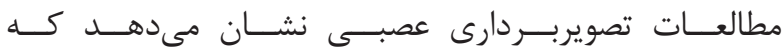

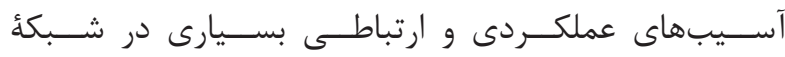

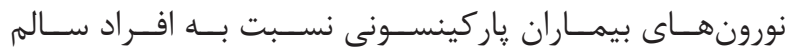

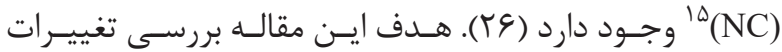

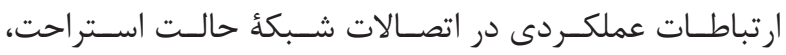

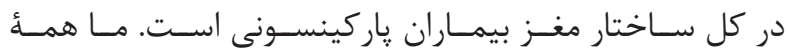

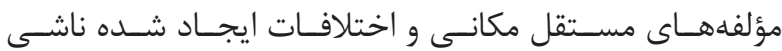

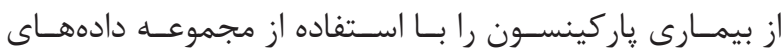

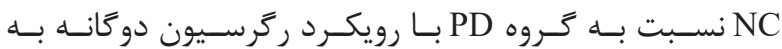
دسـت آوردهايـم.

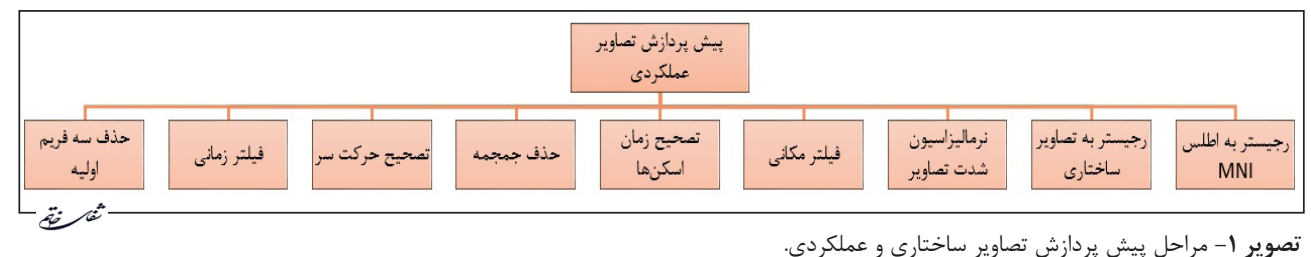

\footnotetext{
${ }^{12}$ Probabilistic ICA

${ }^{13}$ Dual regression

${ }^{14}$ Randomise

${ }^{15}$ Normal control
}

${ }^{16}$ Hoehn and Yahr

${ }^{17}$ Full width half maximum

${ }^{18}$ Montreal neurological institute

${ }^{19}$ Functional magnetic resonance imaging of the brain 


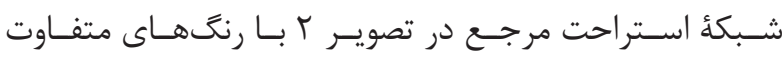

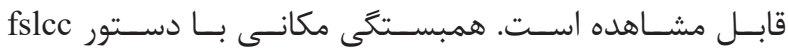

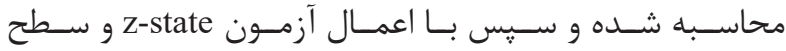

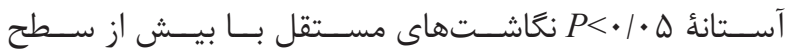

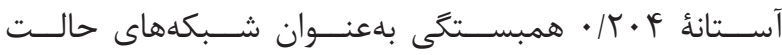

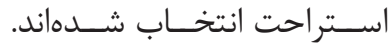
رَّرسيون دوَانه

بــراى تشـخيص شـــكة متفــاوت ارتباطـات بيـن افـراد ســالم

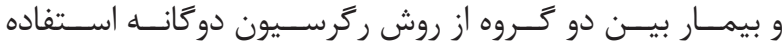

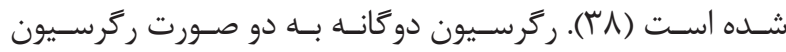

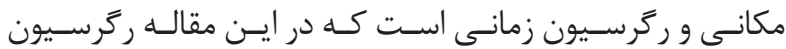

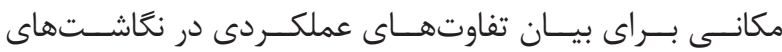

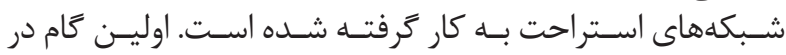

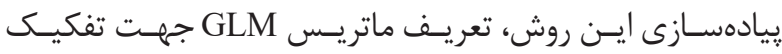

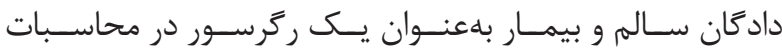

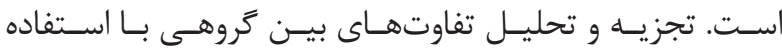

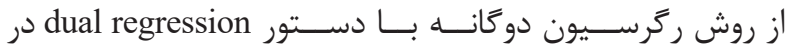

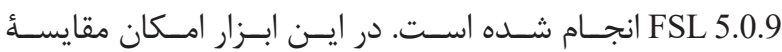

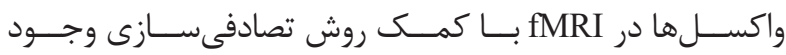

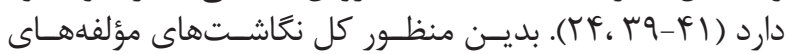

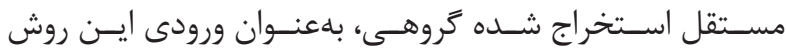

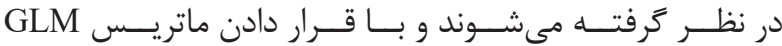

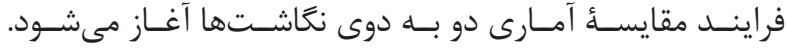

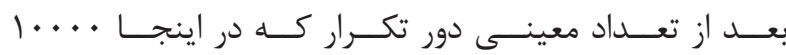

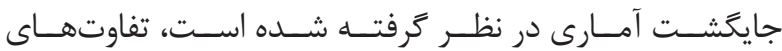

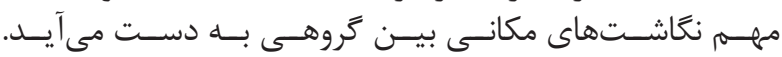

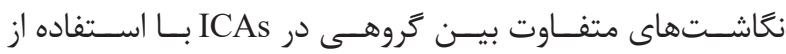

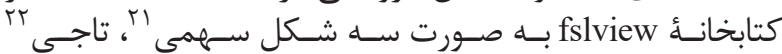

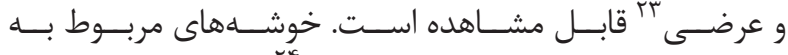

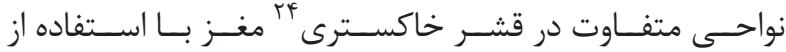

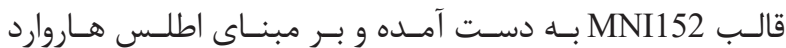

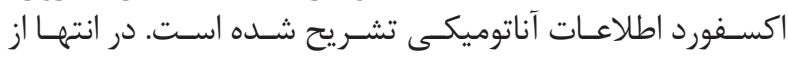

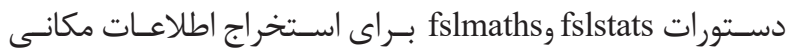

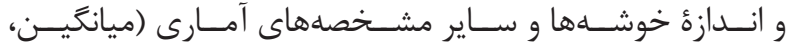

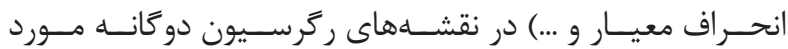

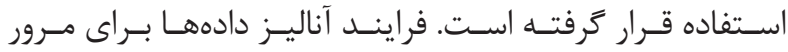

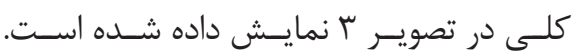

استخراج مؤلفههاى مستقل

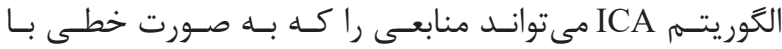

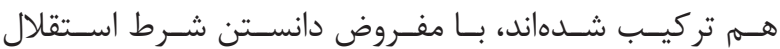

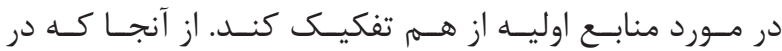

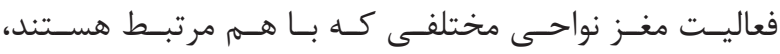

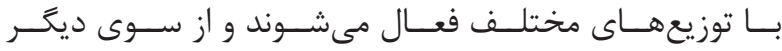

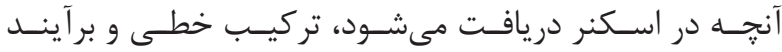

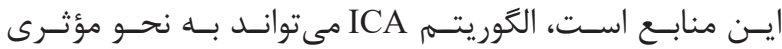

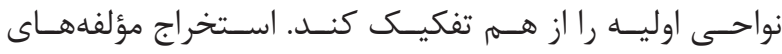

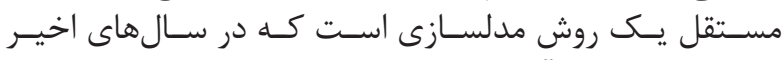

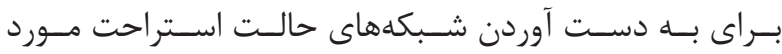

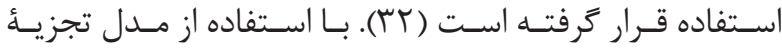

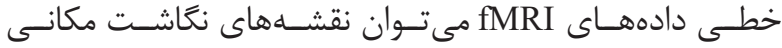

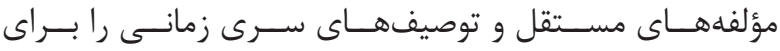

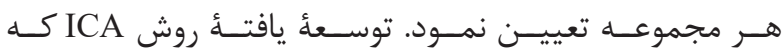

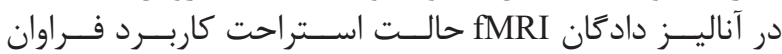

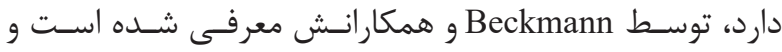

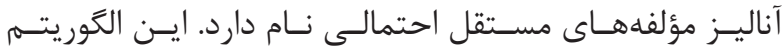

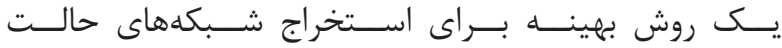

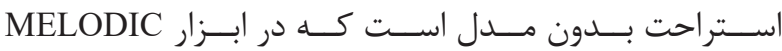

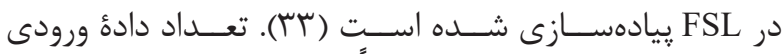
ICA

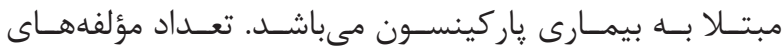

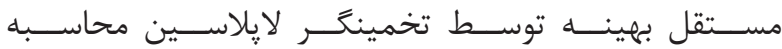

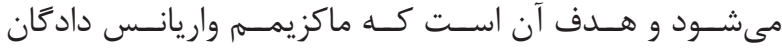

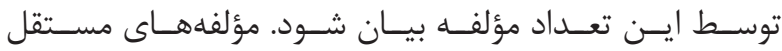

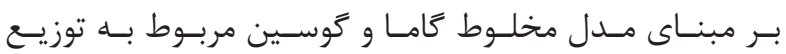

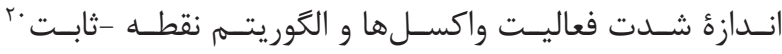

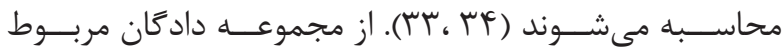

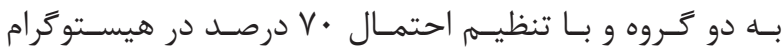

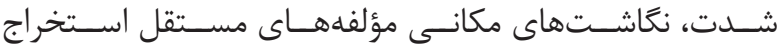

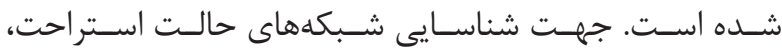

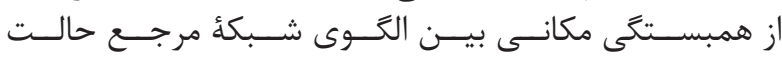

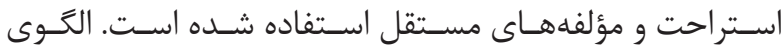

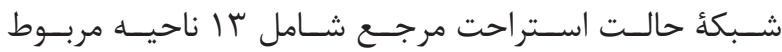

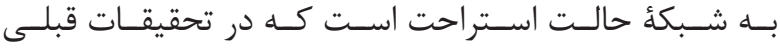

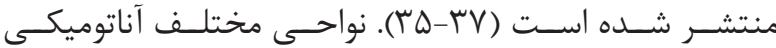

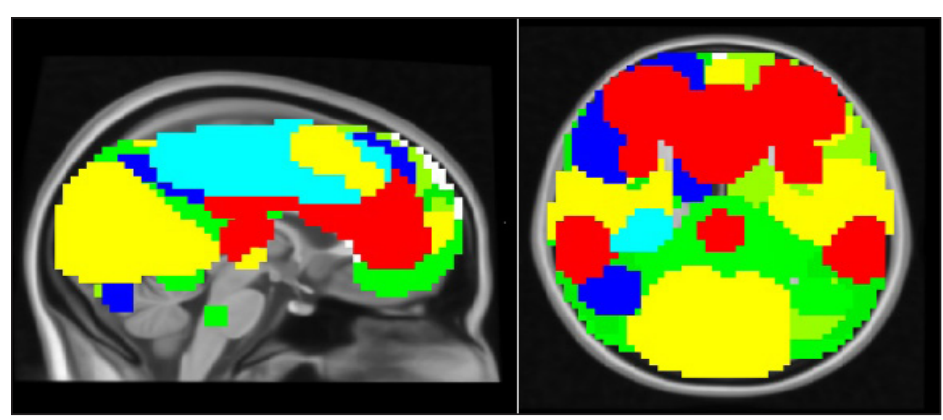

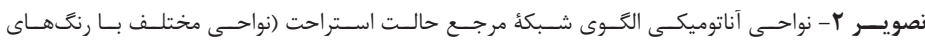

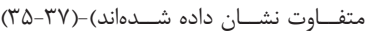

${ }^{20}$ Fixed-point

${ }^{21}$ Sagittal

22 Vertical
${ }^{23}$ Frontal

${ }^{24}$ Gray matter 


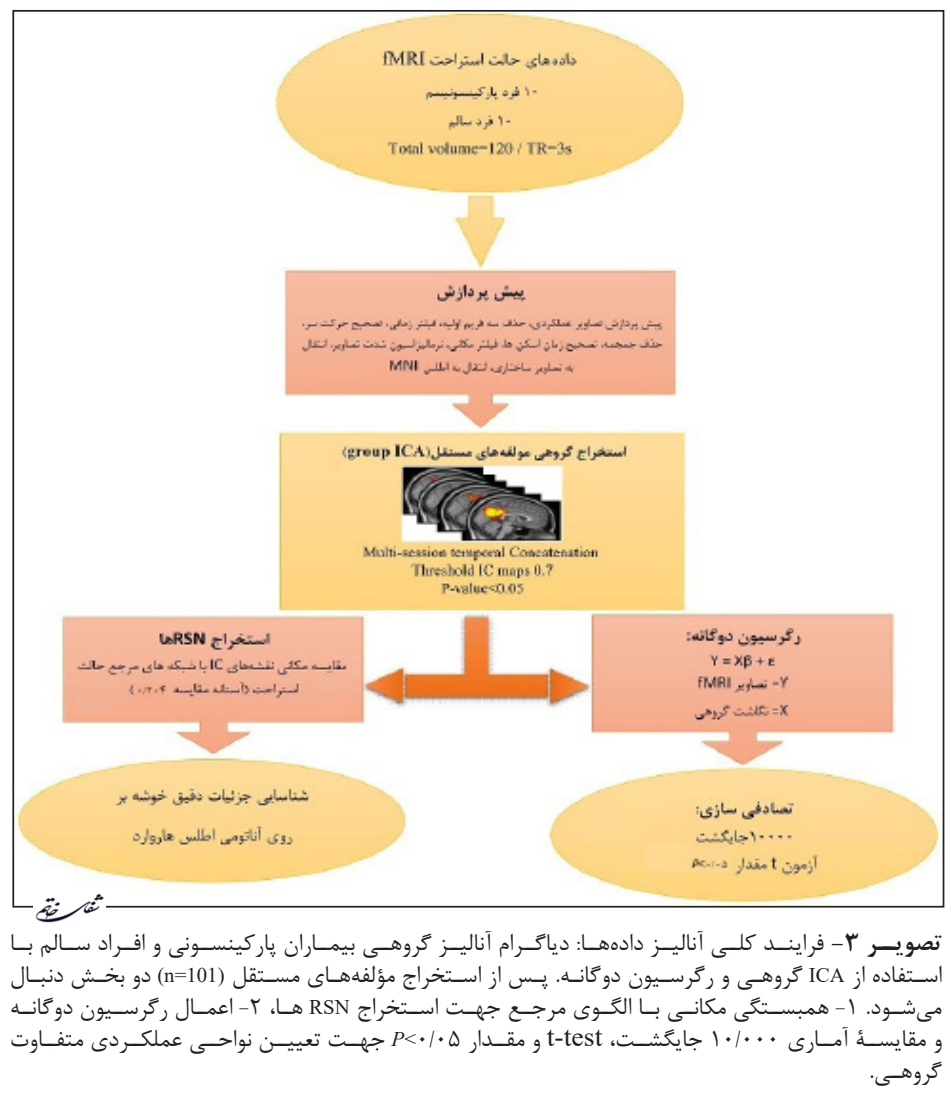

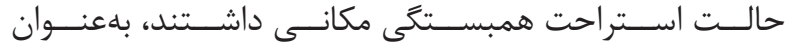

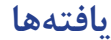

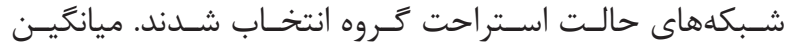

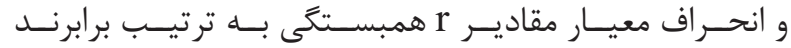

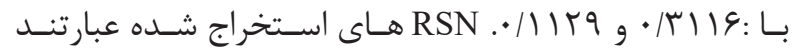

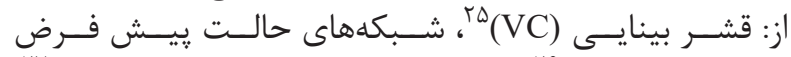

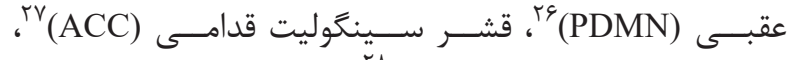

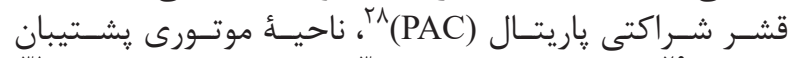

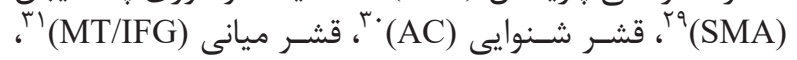

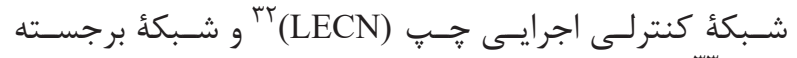
هـ RSN (SN)

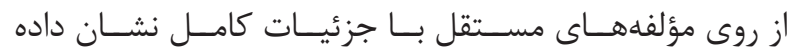

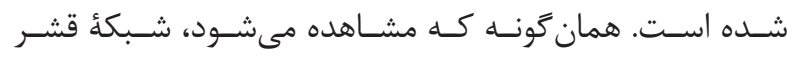

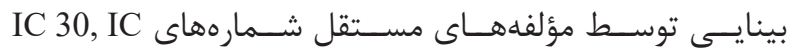
32, IC 57

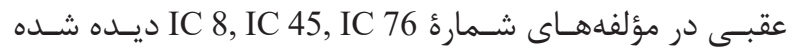

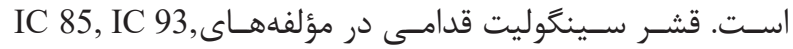

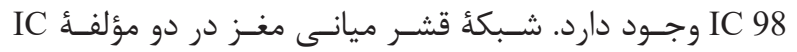

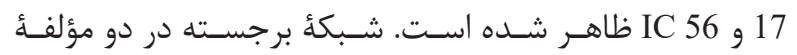

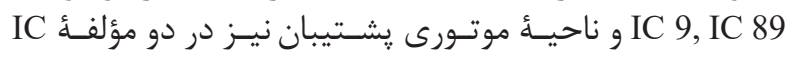

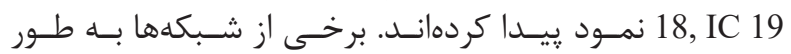

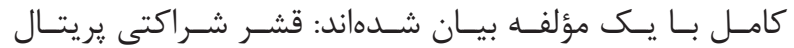

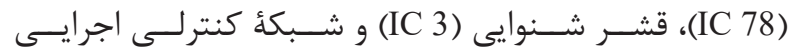

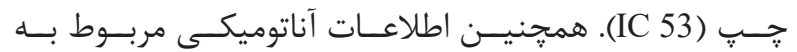

\footnotetext{
${ }^{25}$ Visual cortex

${ }^{26}$ Posterior default mode network

${ }^{27}$ Anterior cingulate/precun cortex

${ }^{28}$ Parietal association cortex

${ }^{29}$ Supplementary motor area
}

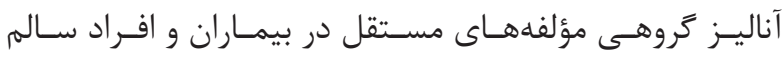

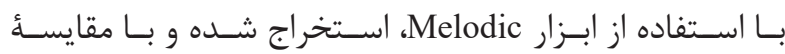

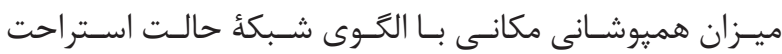

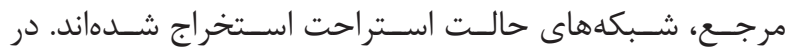

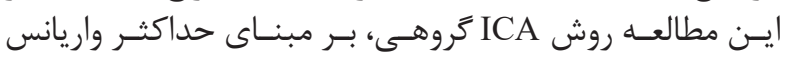

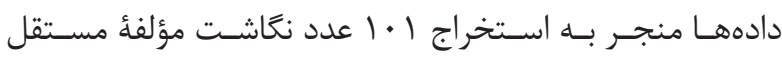

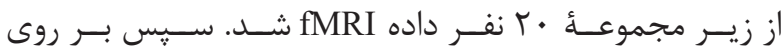

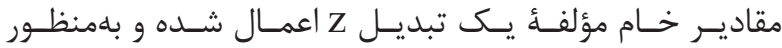

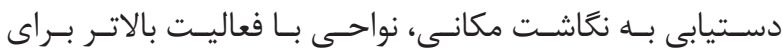

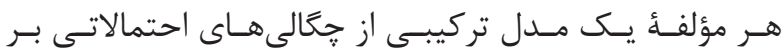

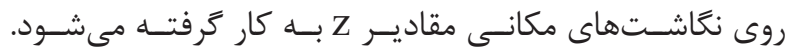

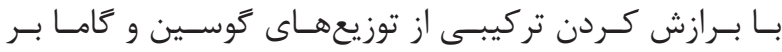

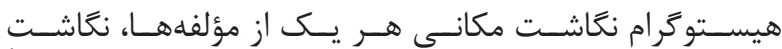

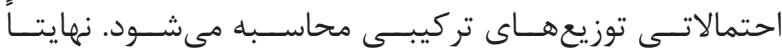

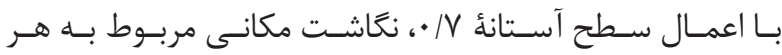

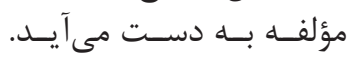

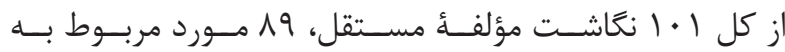

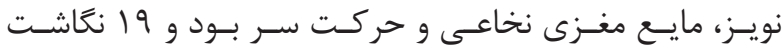

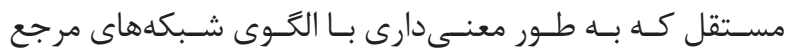

\footnotetext{
${ }^{30}$ Auditory cortex

${ }^{31}$ Middle temporal/IFG

${ }^{32}$ Left executive control network

${ }^{33}$ Salience network
} 


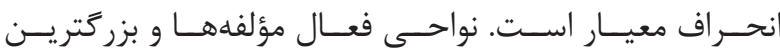

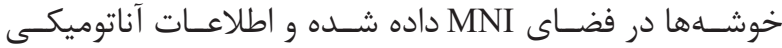

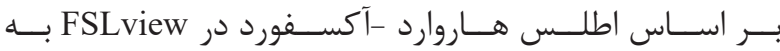

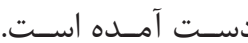

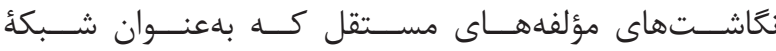

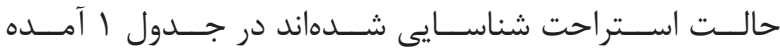

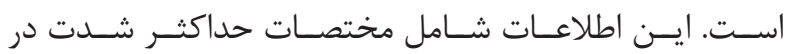

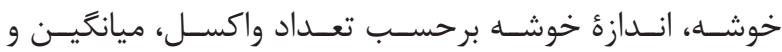

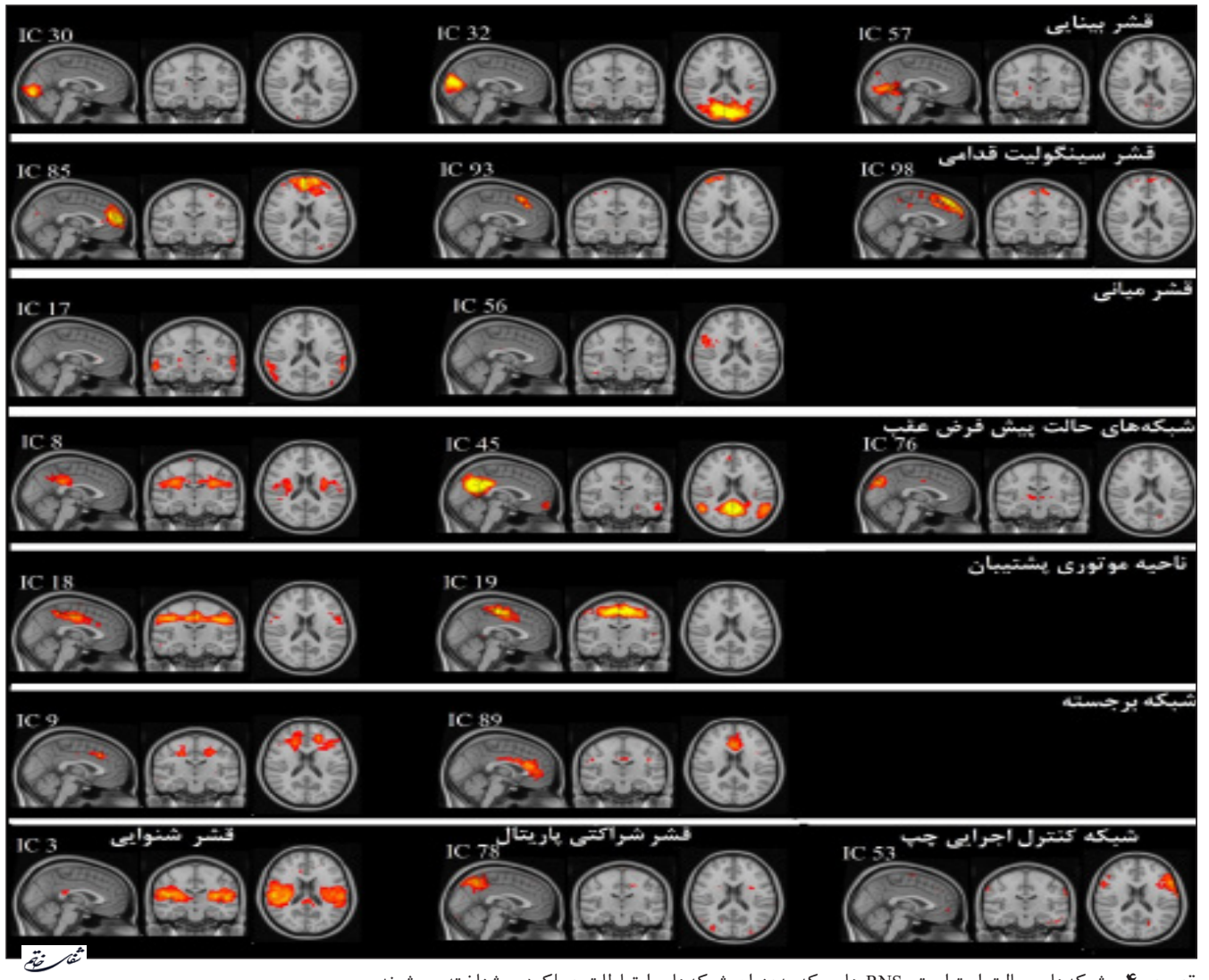

تصوير F - شبكههاى حالت استراحت، RNS هايى كه بلعنوان شبكههاى ارتباطات عملكردى شناخته مىشوند.

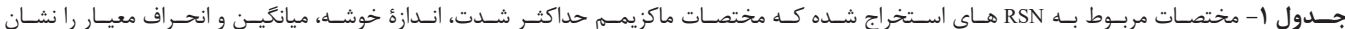

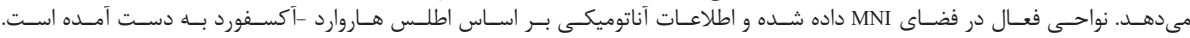

\begin{tabular}{|c|c|c|c|c|c|c|c|c|}
\hline \multirow[t]{2}{*}{ انعر اض معيار } & \multirow[t]{2}{*}{ ميانكين } & \multicolumn{3}{|c|}{ هداكثات مكاتى شدت } & \multirow{2}{*}{ شداحثر } & \multirow[t]{2}{*}{ واكسل } & \multirow[t]{2}{*}{ شبكعباى حالت استراحت } & \multirow[t]{2}{*}{ IC } \\
\hline & & $\mathbf{X}$ & $\mathbf{Y}$ & $\mathbf{Z}$ & & & & \\
\hline$+1+9+1$ & $+l+\mathrm{VV}$ & $r r$ & $D Q$ & Pr & 4/VA & $\Lambda \Delta Y$. & ستر عمقى عركزي، لمشتر رانست متز & $r$ \\
\hline $.15 \times 9$ & $\cdot 1 \cdot A f$ & $\Delta A$ & 18 & $\Delta r$ & $11 / T$ & IVITS & جين سينّولت مغز ، بهش خلفي، قشر راست مغز & $A$ \\
\hline$+\int Y Y Y$ & $+1+19$ & $r \Delta$ & $\mathrm{Vr}$ & $\Delta P$ & $11 / \%$ & $199 \mathrm{rI}$ & إحين سينكولت منز، بخش قداعى، لَشر حي منز & 9 \\
\hline - /. YNT & $* \%+\mathrm{V}$ & 19 & $\mathrm{Fr}$ & 79 & Ir/a & $Y+F G$ & 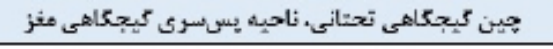 & IV \\
\hline.$/ \Delta \gamma$ & $\cdot 1 \cdot V r$ & Yq & $\Delta p$ & $\Delta f$ & A/rs & $1 \Delta \Delta \& 9$ & 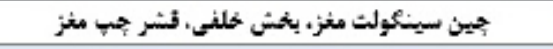 & in \\
\hline -IYGA & $+j+91$ & $\Delta r$ & $\Delta A$ & $g r$ & IF/A & IEFF. & هين سينوسى يبش مركزى مغز. قشر جي مغز & 19 \\
\hline $.19 \times 9$ &.$/ \times \mathrm{VS}$ & $r F$ & 19 & $r s$ & $1 \Delta / r$ & IrYTs & 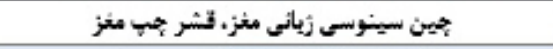 & $r$. \\
\hline AGTF & + & Q. & rr & FV & $1 T / V$ & $1+\wedge \wedge 9$ & قشر فوق كالكارين "م قشر جمي عنز & $r r$ \\
\hline$+/ Y Y I$ & $-(+1)$ & Fq & rr & $P q$ & $19 / 9$ & $\| Y \Delta F$ & 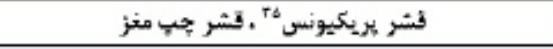 & $P \Delta$ \\
\hline.$/ F F \Delta$ & $* / *$ & $V_{T}$ & VQ & $T \Delta$ & $1 \cdot 1 \mathrm{~A}$ & YIFF & جين جلويى تحتاتى ، زيريخش إيركولاريس. قشر جي مثز & $\Delta r$ \\
\hline +194r & $\cdot 1 \cdot r A$ & $r$. & vr & $r g$ & $19 / 9$ & FVPF & لقشر ععلكردى جلويى، قشر راست منز & $\Delta F$ \\
\hline$\cdot \Delta \Delta \mathbb{F}$ & $* / \Delta 1$ & rr & ro & $\Psi A$ & $1+/ A$ & $9 \Delta 19$ & قيُر زيانى & $\Delta V$ \\
\hline - irat & $+1+\mu \mathrm{F}$ & rv & $r \Delta$ & $\Delta A$ & $11 / 4$ & $\left.\Delta \mathrm{VA}\right|^{+}$ & قشر يريكيونس، لقشر راست مغز & Ve \\
\hline$+1 \Delta+F$ & $\cdot 1 \cdot \Delta A$ & FF & rr & GF & $9 / V \xi$ & IITAY & قشر يريكيونس.قشر جي مغز & $\mathrm{YA}$ \\
\hline.$|F>|$ & $*|\cdot|+\mid$ & $\mathrm{FP}$ & ar & f) & $1 \pi / 9$ & FADF & جيئ هار اسينعولت مغز، قشر حب مغز & $\lambda \Delta$ \\
\hline - ITAT & $+\beta+\gamma+$ & FF & $V A$ & FT & A/IF & rAFI & هين سينكولت. قشر جي هغز & 19 \\
\hline - IAYP &.$/ . \$ \mu$ & TA & $V r$ & \$1 & $1 \pi / 2$ & VDQ9 & جين باراسينبولت مغز، قشر حب مغز & Q4 \\
\hline$\because \Delta \cdot r$ &.$/ . P r$ & PT & $A D$ & 8. & $\mid \Delta / A$ & FAIF & جين جلويى فوفانى. قطب جلويى & $9 A$ \\
\hline
\end{tabular}

${ }^{34}$ Calcarine

${ }^{35}$ Precuneous 


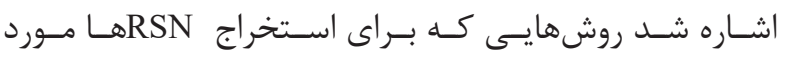

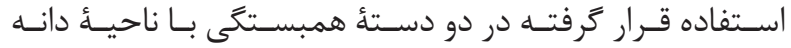

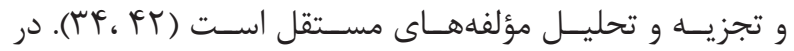

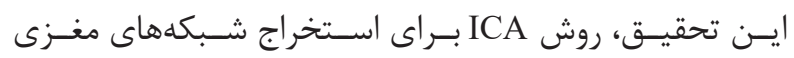

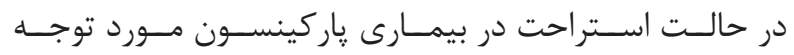

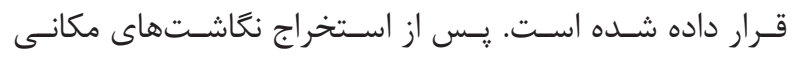

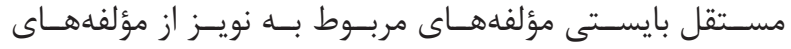

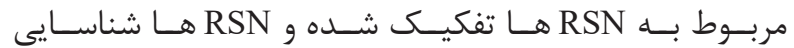

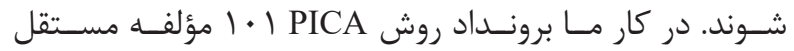

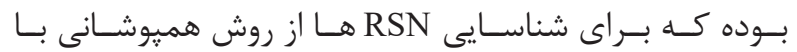

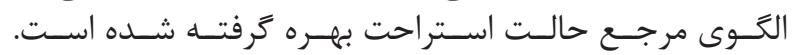

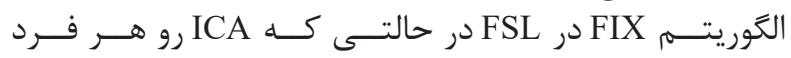

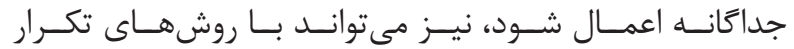

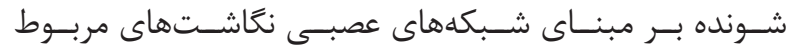

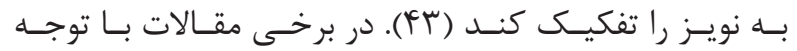

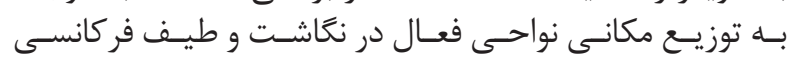

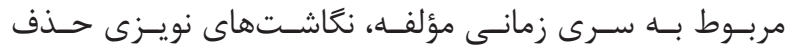

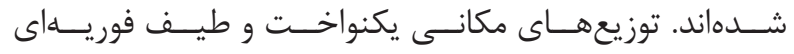

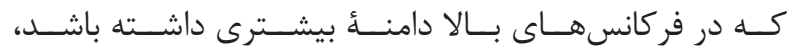

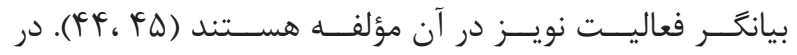

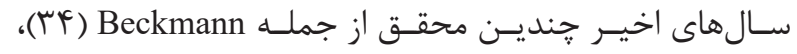
(T) Smith

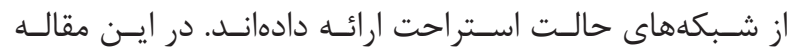

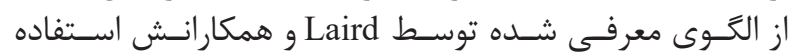

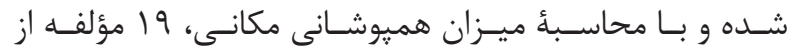

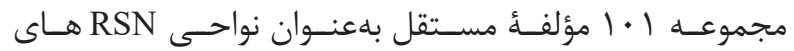

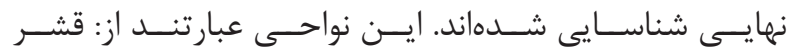

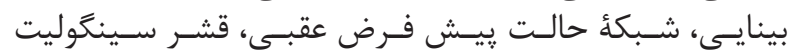

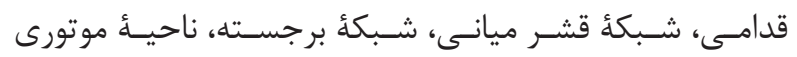

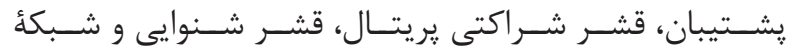

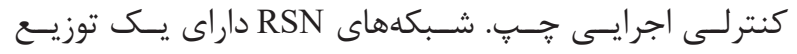

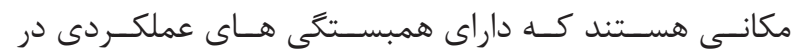

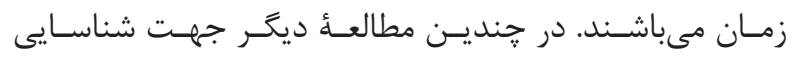

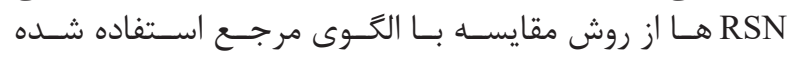

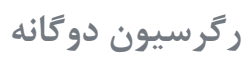

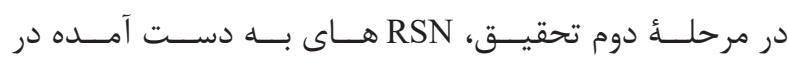

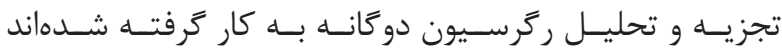

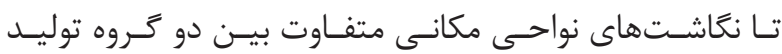

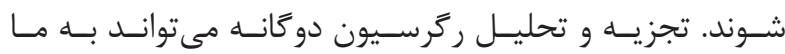

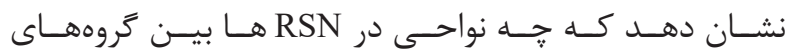

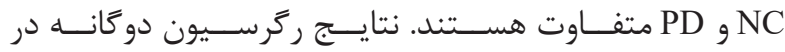

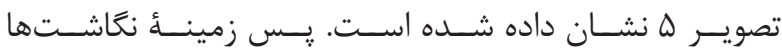

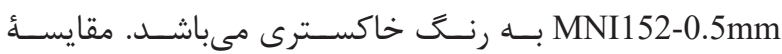

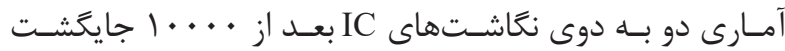

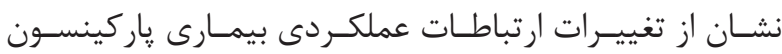

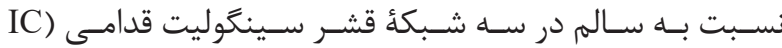

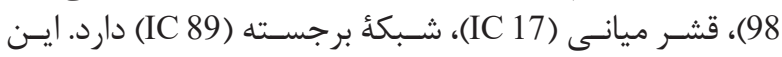

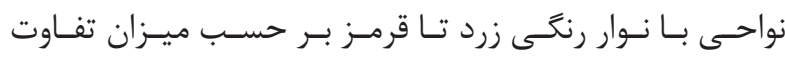

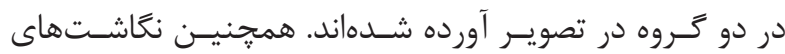

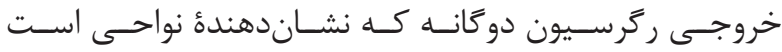

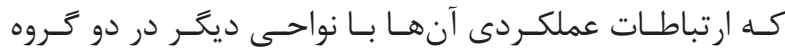

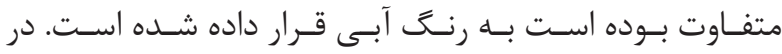

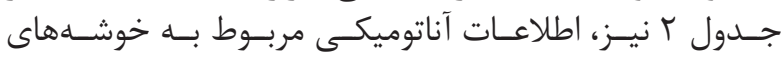

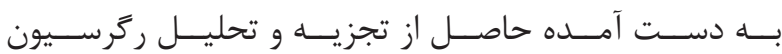

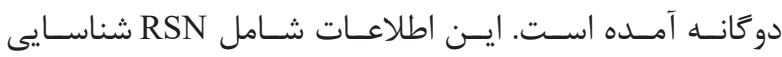

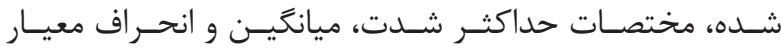

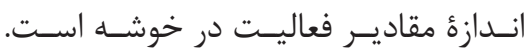

$$
\text { بحث و نتيجه تيرى }
$$

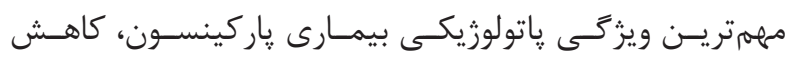

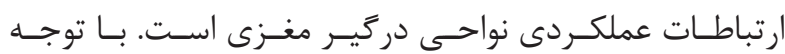

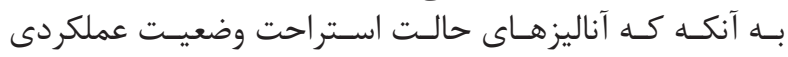

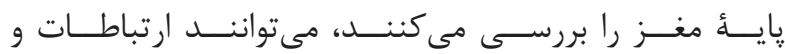

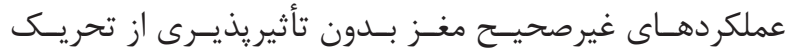

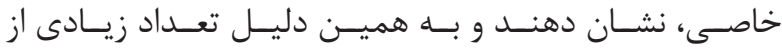

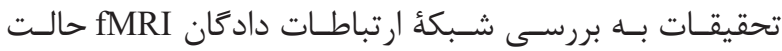

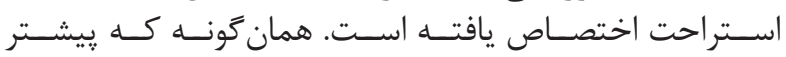

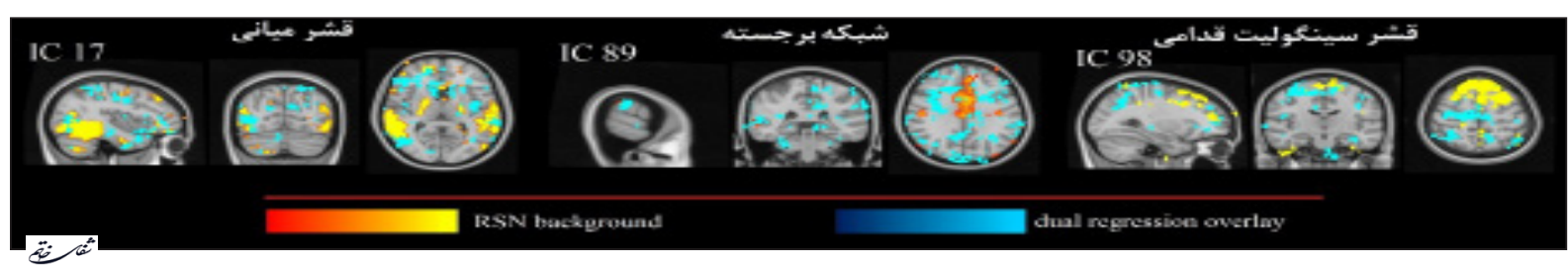

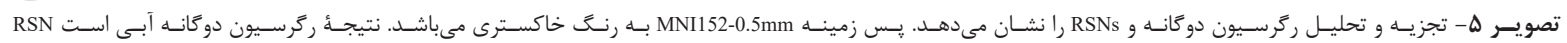

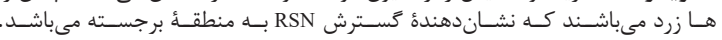

\begin{tabular}{|c|c|c|c|c|c|c|c|c|}
\hline \multirow{2}{*}{ سطح معنى دارى } & \multirow{2}{*}{ انحراف معيار } & \multirow{2}{*}{ ميانكين } & \multicolumn{3}{|c|}{ حداكثر فعاليت مكان } & \multirow{2}{*}{ حداكثر شدت } & \multirow{2}{*}{ شبكههاى حالت استراحت } & \multirow{2}{*}{ IC } \\
\hline & & & $\mathbf{X}$ & $\mathbf{Y}$ & $\mathbf{Z}$ & & & \\
\hline$\cdot 1 \cdot+A$ & $\cdot 1+|V|$ & $\cdot|\cdot| \Delta \mid$ & rV & F. & to & $\cdot / 9 \Delta V$ & قشر ميانى & IV \\
\hline$\cdot 1 \cdot r \Delta$ & $\cdot 1 \cdot 199$ & $\bullet \cdot \cdot v \mathrm{~V}$ & sp & $\Delta \Delta$ & FV &.$/ 9 r$ & شبكه بر جسته & A9 \\
\hline$\because /+F \wedge T$ & $+1+* 999$ & $+1+\cdots+10$ & $\Delta \Delta$ & FF & $r+$ & ./9QT & قشر سينـوليت قدامى & $9 A$ \\
\hline
\end{tabular}

جدول r- مناطق ارتباطى عملكردى متفاوت بين دو گروه در رگرسيون دو گانه را نشان مىدهد. 


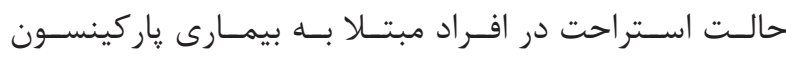

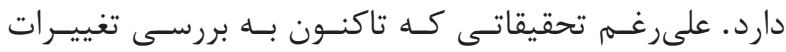

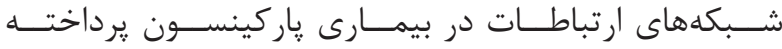

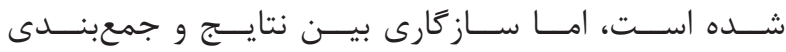

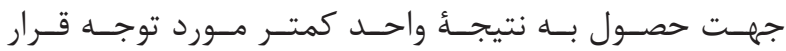

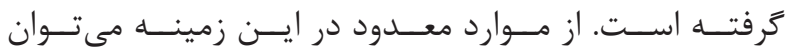

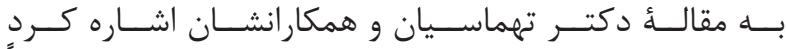

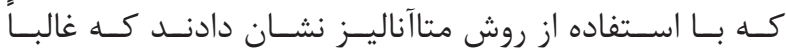

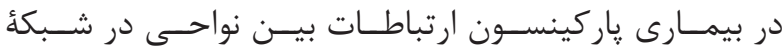
DMN

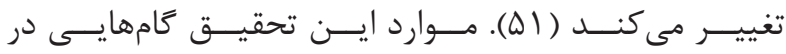

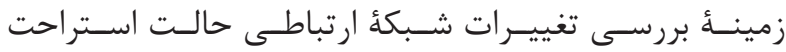

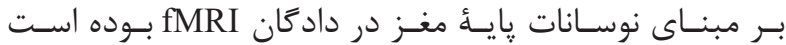

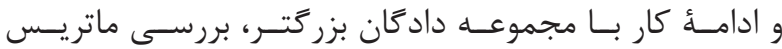

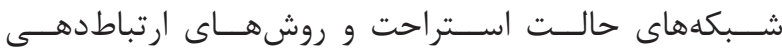

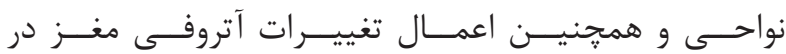

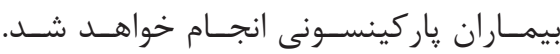
تشكر و قدردانى

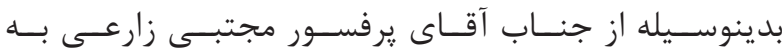

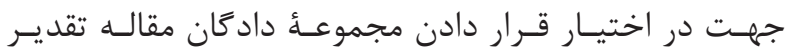

$$
\text { و تشــكر مى شــــود. }
$$

1. Jankovic J. Parkinson's disease: clinical features and diagnosis. J Neurol Neurosurg Psychiatry. 2008; 79(4): 368-76.

2. Sveinbjornsdottir S. The clinical symptoms of Parkinson's disease. J Neurochem. 2016; 139(1): 318-24.

3. Biswal BB. Resting state fMRI: a personal history. Neuroimage. 2012; 62(2): 938-44.

4. Buckner RL, Krienen FM, Yeo BT. Opportunities and limitations of intrinsic functional connectivity MRI. Nat Neurosci. 2013; 16(7): 832-7.

5. Greicius MD, Krasnow B, Reiss AL, Menon V. Functional connectivity in the resting brain: a network analysis of the default mode hypothesis. Proc Natl Acad Sci U S A. 2003; 100(1): 253-8.

6. Fox MD, Raichle ME. Spontaneous fluctuations in brain activity observed with functional magnetic resonance imaging. Nat Rev Neurosci. 2007; 8(9): 700-11.

7. Raichle ME. The brain's default mode network. Annu Rev Neurosci. 2015; 38: 433-47.

8. Agosta F, Pievani M, Geroldi C, Copetti M, Frisoni GB, Filippi M. Resting state fMRI in Alzheimer's disease: beyond the default mode network. Neurobiol Aging. 2012; 33(8): 1564-78.

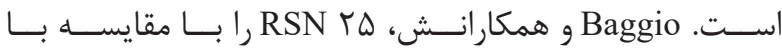

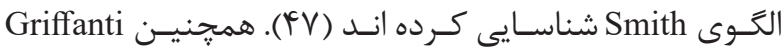

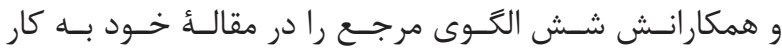

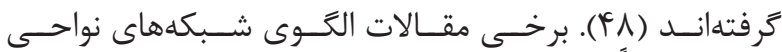

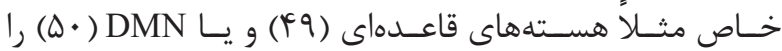

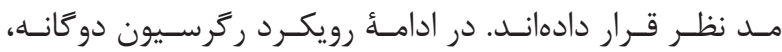

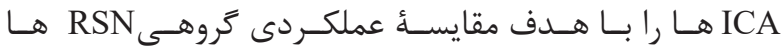

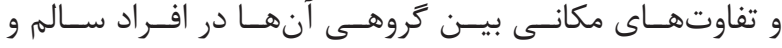

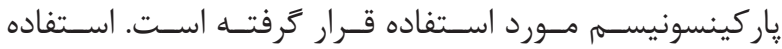

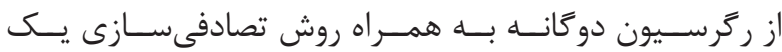

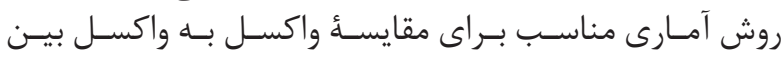

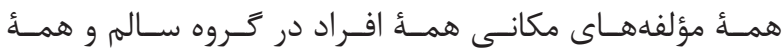

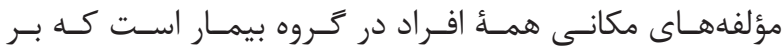

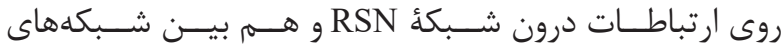

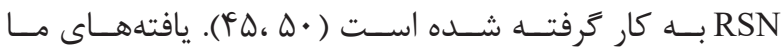

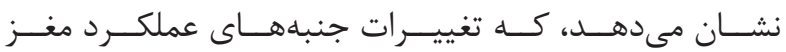

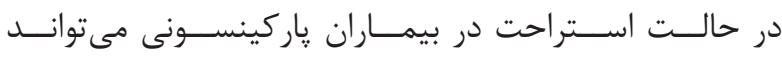

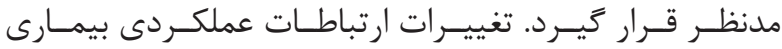

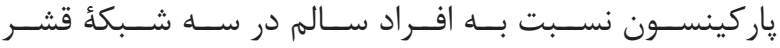

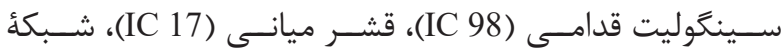

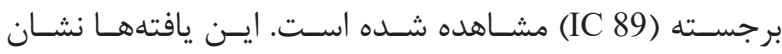

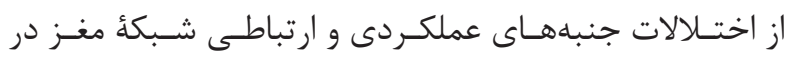

منابع

9. Buckner RL, Andrews-Hanna JR, Schacter DL. The brain's default network: anatomy, function, and relevance to disease. Ann N Y Acad Sci. 2008; 1124(1): 1-38.

10. Greicius MD, Srivastava G, Reiss AL, Menon V. Default-mode network activity distinguishes Alzheimer's disease from healthy aging: evidence from functional MRI. Proc Natl Acad Sci U S A. 2004; 101(13): 4637-42.

11. Rombouts SA, Barkhof F, Goekoop R, Stam CJ, Scheltens P. Altered resting state networks in mild cognitive impairment and mild Alzheimer's disease: an fMRI study. Hum Brain Mapp. 2005; 26(4): 231-9.

12. van Eimeren T, Monchi O, Ballanger B, Strafella AP. Dysfunction of the default mode network in Parkinson disease: a functional magnetic resonance imaging study. Arch Neurol. 2009; 66(7): 877-83.

13. Putcha D, Ross RS, Cronin-Golomb A, Janes AC, Stern CE. Salience and default mode network coupling predicts cognition in aging and Parkinson's Disease. J Int Neuropsychol Soc. 2016; 22(2): 205-15.

14. Gorges M, Müller H-P, Lulé D, Pinkhardt EH, Ludolph AC, Kassubek J, et al. To rise and to fall: functional connectivity in cognitively normal and cognitively impaired patients with Parkinson's disease. Neurobiol Aging. 2015; 36(4): 1727-35. 
15. Tahmasian M, Pasquini L, Scherr M, Meng C, Förster S, Bratec SM, et al. The lower hippocampus global connectivity, the higher its local metabolism in Alzheimer disease. Neurology. 2015; 84(19): 1956-63.

16. Whitwell JL, Przybelski SA, Weigand SD, Ivnik RJ, Vemuri P, Gunter JL, et al. Distinct anatomical subtypes of the behavioural variant of frontotemporal dementia: a cluster analysis study. Brain. 2009; 132(11): 2932-46.

17. Zhou J, Greicius MD, Gennatas ED, Growdon ME, Jang JY, Rabinovici GD, et al. Divergent network connectivity changes in behavioural variant frontotemporal dementia and Alzheimer's disease. Brain. 2010; 133(5): 1352-67.

18. Tessitore A, Esposito F, Vitale C, Santangelo G, Amboni M, Russo A, et al. Default-mode network connectivity in cognitively unimpaired patients with Parkinson disease. Neurology. 2012; 79(23): 2226-32.

19. Disbrow E, Carmichael O, He J, Lanni K, Dressler $\mathrm{E}$, Zhang L, et al. Resting state functional connectivity is associated with cognitive dysfunction in non-demented people with Parkinson's disease. J Parkinsons Dis. 2014; 4(3): 453-65.

20. Wu T, Wang L, Chen Y, Zhao C, Li K, Chan P. Changes of functional connectivity of the motor network in the resting state in Parkinson's disease. Neurosci Lett. 2009; 460(1): 6-10.

21. Amboni M, Tessitore A, Esposito F, Santangelo G, Picillo M, Vitale C, et al. Resting-state functional connectivity associated with mild cognitive impairment in Parkinson's disease. J Neurol. 2015; 262(2): 425-34.

22. Onu M, Badea L, Roceanu A, Tivarus M, Bajenaru O. Increased connectivity between sensorimotor and attentional areas in Parkinson's disease. Neuroradiology. 2015; 57(9): 957-68.

23. Smith SM, Fox PT, Miller KL, Glahn DC, Fox PM, Mackay CE, et al. Correspondence of the brain's functional architecture during activation and rest. Proc Natl Acad Sci U S A. 2009; 106(31): 13040-5.

24. Filippini N, MacIntosh BJ, Hough MG, Goodwin GM, Frisoni GB, Smith SM, et al. Distinct patterns of brain activity in young carriers of the APOE- $\varepsilon 4$ allele. Proc Natl Acad Sci U S A. 2009; 106(17): 7209-14.

25. Zuo X-N, Kelly C, Adelstein JS, Klein DF, Castellanos FX, Milham MP. Reliable intrinsic connectivity networks: test-retest evaluation using ICA and dual regression approach. Neuroimage. 2010; 49(3): 2163-77.
26. Jubault T, Brambati SM, Degroot C, Kullmann B, Strafella AP, Lafontaine A-L, et al. Regional brain stem atrophy in idiopathic Parkinson's disease detected by anatomical MRI. PloS one. 2009; 4(12): e8247.

27. Goetz CG, Poewe W, Rascol O, Sampaio C, Stebbins GT, Counsell C, et al. Movement disorder society task force report on the hoehn and yahr staging scale: status and recommendations the movement disorder society task force on rating scales for Parkinson's disease. Mov Disord. 2004; 19(9): 1020-8.

28. Hoehn MM. Melvin D Yahr. Parkinson: onset, progresión, and mortality. Neurology. 1967; 17: 427-42.

29. Helmich RC, Derikx LC, Bakker M, Scheeringa R, Bloem BR, Toni I. Spatial remapping of cortico-striatal connectivity in Parkinson's disease. Cerebral Cortex. 2009; 20(5): 1175-86.

30. Smith SM. Fast robust automated brain extraction. Hum Brain Mapp. 2002; 17(3): 143-55.

31. Rueckert D, Sonoda LI, Hayes C, Hill DL, Leach MO, Hawkes DJ. Nonrigid registration using free-form deformations: application to breast MR images. IEEE Transactions on Medical Imaging. 1999; 18(8): 712-21.

32. Abou Elseoud A, Littow H, Remes J, Starck T, Nikkinen J, Nissilä $\mathrm{J}$, et al. Group-ICA model order highlights patterns of functional brain connectivity. Front Syst Neurosci. 2011; 5: 37. doi.org/10.3389/ fnsys.2011.00037.

33. Beckmann CF, Smith SM. Probabilistic independent component analysis for functional magnetic resonance imaging. IEEE Transactions on Medical Imaging. 2004; 23(2): 137-52.

34. Beckmann CF, DeLuca M, Devlin JT, Smith SM. Investigations into resting-state connectivity using independent component analysis. Philosophical Transactions of the Royal Society B: Biological Sciences. 2005; 360(1457): 1001-13.

35. Calhoun VD, Adali T, Pearlson GD, Pekar J. A method for making group inferences from functional MRI data using independent component analysis. Hum Brain Mapp. 2001; 14(3): 140-51.

36. Chang C, Glover GH. Effects of model-based physiological noise correction on default mode network anti-correlations and correlations. Neuroimage. 2009; 47(4): 1448-59.

37. Thomason ME, Dennis EL, Joshi AA, Joshi SH, Dinov ID, Chang C, et al. Resting-state fMRI can 
reliably map neural networks in children. Neuroimage. 2011; 55(1): 165-75.

38. Kiviniemi V, Starck T, Remes J, Long X, Nikkinen J, Haapea M, et al. Functional segmentation of the brain cortex using high model order group PICA. Hum Brain Mapp. 2009; 30(12): 3865-86.

39. Abou-Elseoud A, Starck T, Remes J, Nikkinen J, Tervonen O, Kiviniemi V. The effect of model order selection in group PICA. Hum Brain Mapp. 2010; 31(8): 1207-16.

40. Littow H, Abou Elseoud A, Haapea M, Isohanni M, Moilanen I, Mankinen K, et al. Age-related differences in functional nodes of the brain cortex-a high model order group ICA study. Front Syst Neurosci. 2010; 4: 32. doi: $10.3389 /$ fnsys.2010.00032.

41. Veer IM, Beckmann C, Van Tol M-J, Ferrarini L, Milles J, Veltman D, et al. Whole brain resting-state analysis reveals decreased functional connectivity in major depression. Front Syst Neurosci. 2010; 4: 41. doi: 10.3389/fnsys.2010.00041.

42. Smith DV, Utevsky AV, Bland AR, Clement N, Clithero JA, Harsch AE, et al. Characterizing individual differences in functional connectivity using dualregression and seed-based approaches. Neuroimage. 2014; 95: 1-12.

43. Salimi-Khorshidi G, Douaud G, Beckmann CF, Glasser MF, Griffanti L, Smith SM. Automatic denoising of functional MRI data: combining independent component analysis and hierarchical fusion of classifiers. Neuroimage. 2014; 90: 449-68.

44. Griffanti L, Douaud G, Bijsterbosch J, Evangelisti S, Alfaro-Almagro F, Glasser MF, et al. Hand classification of fMRI ICA noise components. Neuroimage. 2017;
154: 188-205.

45. Peraza LR, Nesbitt D, Lawson RA, Duncan GW, Yarnall AJ, Khoo TK, et al. Intra-and inter-network functional alterations in $\mathrm{P}$ arkinson's disease with mild cognitive impairment. Hum Brain Mapp. 2017; 38(3): 1702-15.

46. Laird AR, Fox PM, Eickhoff SB, Turner JA, Ray KL, McKay DR, et al. Behavioral interpretations of intrinsic connectivity networks. J Cogn Neurosci. 2011; 23(12): 4022-37.

47. Baggio HC, Segura B, Sala-Llonch R, Marti MJ, Valldeoriola F, Compta Y, et al. Cognitive impairment and resting-state network connectivity in Parkinson's disease. Hum Brain Mapp. 2015; 36(1): 199-212.

48. Griffanti L, Rolinski M, Szewczyk-Krolikowski K, Menke RA, Filippini N, Zamboni G, et al. Challenges in the reproducibility of clinical studies with resting state fMRI: An example in early Parkinson's disease. Neuroimage. 2016; 124: 704-13.

49. Rolinski M, Griffanti L, Szewczyk-Krolikowski K, Menke RA, Wilcock GK, Filippini N, et al. Aberrant functional connectivity within the basal ganglia of patients with Parkinson's disease. Neuroimage Clin. 2015; 8: 126-32.

50. Yao N, Shek-Kwan Chang R, Cheung C, Pang S, Lau KK, Suckling J, et al. The default mode network is disrupted in Parkinson's disease with visual hallucinations. Hum Brain Mapp. 2014; 35(11): 5658-66.

51. Tahmasian M, Eickhoff SB, Giehl K, Schwartz F, Herz DM, Drzezga A, et al. Resting-state functional reorganization in Parkinson's disease: An activation likelihood estimation meta-analysis. Cortex. 2017; 92: 119-38. 